\title{
Lattice match: An application to heteroepitaxy
}

\author{
A. Zur and T. C. McGill \\ California Institute of Technology, Pasadena, California 91125
}

(Received 11 July 1983; accepted for publication 20 September 1983)

\begin{abstract}
We define the concept of lattice match for any pair of crystal lattices in any given crystal direction, allowing for a periodic reconstruction of the interface. An algorithm for a systematic search for all possible matches is developed, and some examples of nonstandard lattice matches are given for $\mathrm{CdTe}$ on GaAs and sapphire to illustrate the method. For the case of CdTe on GaAs, our results agree with published results, both with respect to growth plane and orientation for $\mathrm{CdTe}(111)$ on $\mathrm{GaAs}(100)$. For CdTe on sapphire, our results agree with published results with respect to growth plane.
\end{abstract}

PACS numbers: $68.55 .+b, 68.48 .+\mathrm{f}$

\section{INTRODUCTION}

In recent years there has been a growing interest in the epitaxial growth of one material on another. In the past, most of the interesting systems were such that the epitaxial layer had the same crystal structure and the same orientation as that of the substrate. However, there are many important cases where the epitaxial layer has either a different orientation than the substrate [e.g., CdTe $(1,1,1)$ on $\mathrm{GaAs}$ $\left.(1,0,0)^{1}\right]$ or a totally different crystal structure (e.g., silicon on sapphire, ${ }^{2} \mathrm{CdTe}$ on sapphire, ${ }^{3}$ and others). For such systems, the old criterion of lattice match, namely, comparing the lattice parameters, is no longer applicable, and a new criterion should be used. Instead of comparing the bulk lattice parameters, we shall compare the interface translational symmetry with that of the bulk materials on both sides of the interface. We shall define two lattices to match, if the interface translational symmetry could be compatible with the symmetry on both sides of the interface, to within a given precision. To clarify this point, refer to Fig. 1, in which we display the two-dimensional translations parallel to the (101) face of the rhombohedral $\mathrm{Al}_{2} \mathrm{O}_{3}$ (or (1 $\left.1 \overline{1} 02\right)$ in the more com-

SILICON (III)
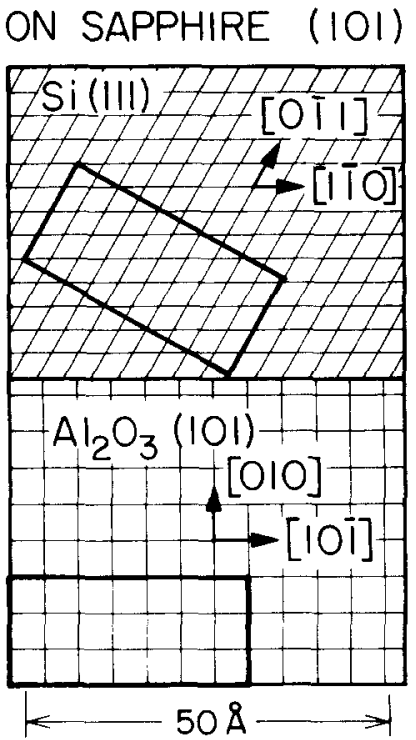

mon hexagonal notation) and the (111) face of the face centered cubic silicon. The lattice parameters of $\mathrm{Al}_{2} \mathrm{O}_{3}$ are $a_{r h}$ $=5.1286 \AA$ and $\alpha=55^{\circ} 17.36^{\prime}{ }^{4}$ Its (101) face has a twodimensional (2D) rectangular symmetry with unit cell edges

$$
2 a \sin (\alpha / 2)=4.759 \text { by } a=5.129 \text {. }
$$

The (111) face of the silicon forms a $120^{\circ}$ rhombic grid, with edges of $\sqrt{1 / 2} a=3.840$. These two-dimensional lattices are depicted by the fine line grids in Fig. 1. One can now form superlattices by taking larger unit cells. If we take 21 sapphire unit cells and 40 silicon unit cells in the manner seen in Fig. 1, we obtain cells that are very similar. The sapphire superlattice cell is rectangular, 15.386 by $33.315 \AA$. The silicon superlattice cell is also rectangular, 15.361 by $33.258 \AA$. The mismatch in each direction is about $0.2 \%$. One can see that the $\mathrm{Al}_{2} \mathrm{O}_{3}[001]$ direction ([$[\overline{1} 101]$ in the hexagonal notation) is parallel to one of the three equivalent $\mathrm{Si}[01 \overline{1}],[10 \overline{1}]$, [110] directions. We do not claim here that this is actually the way silicon (111) grows on sapphire (101). This example serves merely to illustrate the fact that there are some possible orientations of the interface that match to within a high precision.

Lattice match, as defined above, is a very similar idea to the concept of coincidence-site lattice, ${ }^{5}$ that appears to play an important role in high angle grain boundaries. ${ }^{6}$ The main difference between the two is that, in grain boundaries, both sides of the interface consist of the same material, while in heteroepitaxy, this is in general not true. When the same material occupies both sides of the interface, the match across the interface can be exact. When the interface is between two different materials, such an exact match cannot be found, and there will always be some finite mismatch. This finite mismatch forces us to abandon the number-theoretical calculations so popular in coincidence-site lattice theory. ${ }^{7}$ Instead, we give in the next section a different approach, based on a reduction scheme used in Minkowski geometry of numbers. ${ }^{8}$ This approach is similar to the one developed by Santoro and Mighell. ${ }^{9}$

The role of lattice match in epitaxial growth is still not well understood. Lattice match in this context means that the two-dimensional interface possesses translational symmetry that is compatible with that of the bulk on both sides 
of the interface. Such compatibility enables local structures of the interface to repeat themselves periodically over large distances. Actually, the chemistry of the interface will always play a major role as far as growth is concerned. The effect of lattice mismatch will be secondary, resulting in defects, which in turn increase the interface energy. Therefore, a good lattice match is never a sufficient condition for actual growth. We may hope, however, that the converse is true, namely, that certain film faces will not grow as single crystals on a given substrate because of a poor match. It is important at this stage to characterize good or bad lattice matches and to compare them with known experimental results.

We would like to emphasize here that this model covers only sharp, single-crystalline interfaces with a possible periodic reconstruction. Polycrystalline interfaces, stepped interfaces or interfaces in which the atomic reconstruction is not periodic are not covered by this model. We would also like to stress the fact that we did not consider the local chemistry at the interface.

As we have seen in the previous example, the lattice match is characterized by two parameters: the precision of the match and the minimal unit cell area. The common unit cell in Fig. 1 has a mismatch of at most $0.2 \%$ in both lateral dimensions and the angle between them. The unit cell area is $511 \AA^{2}$. This cell is the smallest possible one that will enable a mismatch of less than $1 \%$ in the unit cell sides and angle. One can see that there is a trade-off between the precision of a match and the size of the superlattice cells. If we allow matches with a very large superlattice cell, there are many such cells to be considered, and we increase the odds of finding a more precise match. On the other hand, the larger the size of the interface unit cell, the less likely it is that the chemical forces will reinforce the lattice match condition. So far, we do not have any criterion as to when a match is too poor to allow epitaxial growth. The precision of the match gives a lower bound to changes in lateral interatomic distances. For example, suppose the mismatch in one of the sides of the common unit cell is $1 \%$. In that case, this one percent can be accommodated at best by a lateral movement of $\pm 0.5 \%$ of every atom on both sides of the interface. In reality, not all the atoms will move by the same amount, and there will be some lateral interatomic distance that will change by more than $0.5 \%$. Such changes in interatomic distances will introduce strain in the crystal. Thick epitaxial film will tend to relieve this strain energy by creating defects. The common unit cell area should be limited to a value that is reasonable for interfacial periodic reconstruction. Recalling that the free (111) face of silicon reconstructs spontaneously to form a $(7 \times 7)$ unit cell with an area of $625 \AA^{2}$, we feel that common unit cells of a few hundred square angstroms are still reasonable. In this paper, limits of $1 \%$ on the match precision and $600 \AA^{2}$ on the common unit cell area will be shown to be sufficient to reproduce the experimental results of $\mathrm{CdTe}(111) / \mathrm{GaAs}(100)$ of Cheung $^{1}$ and $\mathrm{CdTe}(111) /$ $\mathrm{Al}_{2} \mathrm{O}_{3}$ of Myers et al. ${ }^{3}$

With the match precision and the common unit cell area taken as parameters, the problem of lattice match is now well defined. A computer can easily scan many possible pairs of materials in various directions and find all the possi- ble good lattice matches. In this way, we can generate a list of interfaces that match to within a prescribed precision, and this list will help us reduce the number of "interesting" interfaces. This reduction comes in very handy when we consider the number of possible interfaces.

This paper is organized in the following way. In Sec. II, we discuss the geometrical principles of lattice match and the systematic way to find matches to within a given precision and size. This will be done in a descriptive rather than rigorous way. Section III illustrates the method by applying it to the $\mathrm{CdTe} / \mathrm{GaAs}$ and $\mathrm{CdTe} /$ sapphire systems. These systems were chosen since recent experimental results of actual heteroepitaxial growth are available. In Sec. IV we draw some conclusions based on this study.

\section{GEOMETRICAL PRINCIPLES OF LATTICE MATCH}

A cut in any crystal direction through a three-dimensional lattice results in a surface which has a $2 \mathrm{D}$ translational symmetry. Any additional reconstruction of the atoms near the interface during the interface formation may, in general, reduce the symmetry of the unreconstructed surface. Thus, the symmetry group of the reconstructed surface will be a subgroup of the symmetry group of the unreconstructed surface. We see, therefore, that the problem of lattice match is reduced to scanning the $2 \mathrm{D}$ cuts in a given pair of lattices, and then comparing the two resulting $2 \mathrm{D}$ lattices, looking for a common superlattice.

The most fundamental question is how to tell when two 2D lattices are actually the same (or nearly the same). The difficulty arises from two sources. First, the two similar lattices may be rotated with respect to each other, or one could be a rotation of a mirror image of the other. In this case, we want to be able to identify the two lattices as being the "same." Second, there is no simple and unique way to describe a lattice. A lattice is uniquely determined once its primitive translations are given. The primitive translations are a set of vectors such that every lattice point can be expressed uniquely as a linear combination of these vectors with integer coefficients. While a set of primitive translations determines the lattice, the converse is not true, and there are an infinite number of possible sets of primitive translations generating a given lattice. We want to be able to identify two such sets as belonging to the same lattice or to the same lattice rotated or reflected. The way to achieve this is by using a reduction scheme. ${ }^{8}$ The reduction process selects a particular set of primitive translations from all the possible ones in almost a unique way. In this process, only intrinsic properties of the lattice, (i.e., lengths and angles) determine the final choice of primitive translations without any reference to a particular coordinate system. Therefore, any rotation or reflection of the lattice, which amounts to a different coordinate system, will not have any influence on the choice of the primitive translations.

To be more specific, given a two-dimensional lattice, a set of primitive translations $(\mathbf{a}, \mathbf{b})$ is called reduced if it satisfies the following three conditions: (a) $a$ is the shortest possible nonzero vector of the lattice; $(b) b$ is the shortest possible lattice vector, linearly independent of $\mathbf{a}$; (c) the angle between $\mathbf{a}$ and $\mathbf{b}$ is nonobtuse. 
One needs a constructive procedure to obtain a reduced translations set from a nonreduced one. Such a procedure is given in the flow chart of Fig. 2. We can see in that figure that the procedure is very simple and amounts to successive additions or substractions of the shorter translation vector from the longer until no further reduction in size of the translation vectors can be achieved. Finally, we reverse the sign of one of the vectors, if the angle between the two vectors is obtuse. The procedure is guaranteed to end after a finite number of steps and to result in a reduced set of translations. Another important feature of this procedure is that it conserves the area of the unit cell $(0, \mathbf{a}, \mathbf{b}, \mathbf{a}+\mathbf{b})$ in every step.

In general, a lattice may have more than one reduced set of primitive translations. However, the lengths of the reduced translation vectors, as well as the angle between them, is unique. These three numbers, namely,

$$
a=|\mathbf{a}|, \quad b=|\mathbf{b}|, \text { and } \cos \alpha=\frac{|\mathbf{a} \cdot \mathbf{b}|}{|\mathbf{a}| \cdot|\mathbf{b}|},
$$

are uniquely determined by the lattice and also uniquely determine the lattice up to an arbitrary rotation or reflection. Thus, the procedure of determining when two lattices are the same, up to an arbitrary rotation, reflection and a choice of

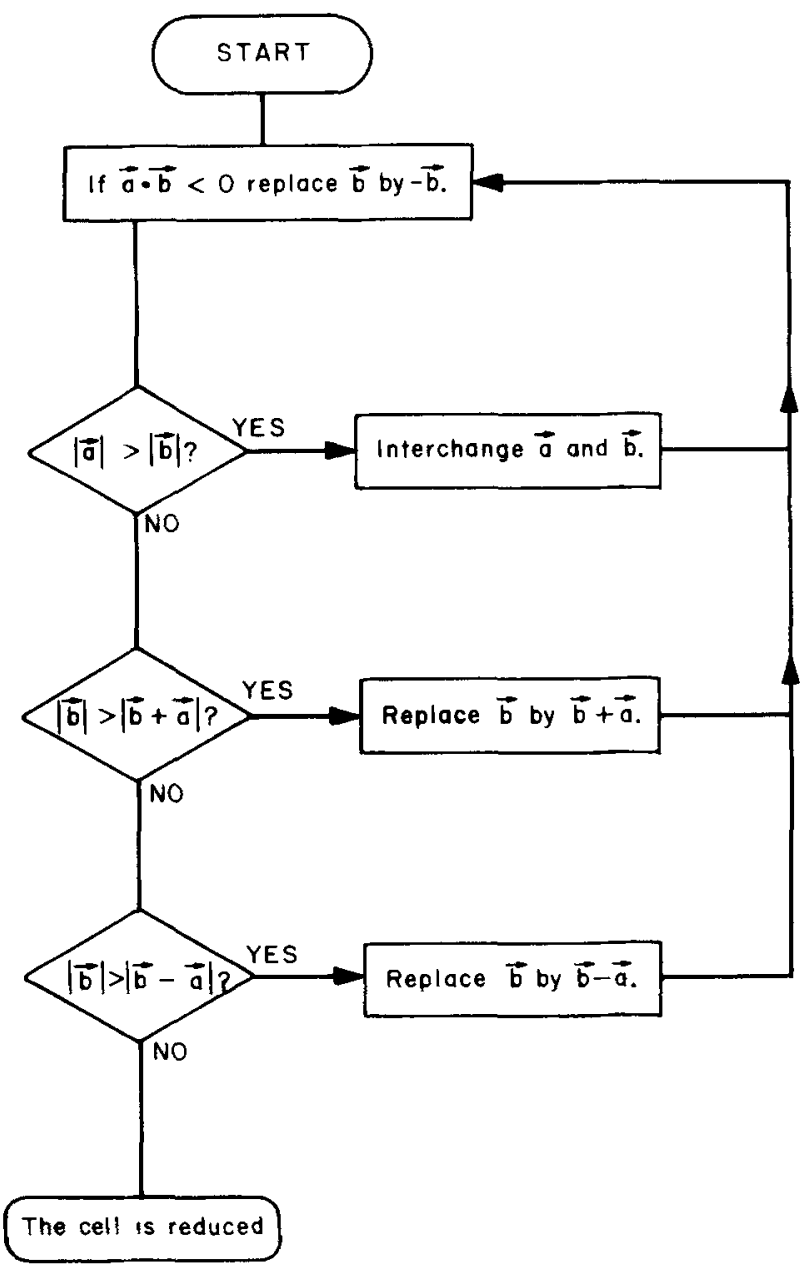

FIG. 2. Flow chart of the unit cell reduction procedure. primitive translations set, is completely solved. One can also compare the $a, b$, and $\alpha$ mentioned above to determine quantitatively how similar two lattices are.

The next problem is to determine when two 2D lattices match. We say that two lattices match, if each one of them possesses a superlattice, such that the two superlattices are the same (up to a rotation, reflection, and a choice of primitive translations). The task of scanning and comparing all possible superlattices of the two lattices looks at the outset to be a formidable one. In fact, it is not that bad. Recall the fact that the unit cell area of a superlattice is an integer times the unit cell area of the original lattice. If the original lattices had unit cells area and $A_{1}$ and $A_{2}$, the superlattices have unit cell areas of $r_{1} A_{1}$ and $r_{2} A_{2}$, respectively, where $r_{1}$ and $r_{2}$ are integers. Since we want the superlattices to be the same, they must have the same unit cell area. Thus $r_{1} A_{1}=r_{2} A_{2}$, or

$$
\frac{r_{1}}{r_{2}}=\frac{A_{2}}{A_{1}} \text {. }
$$

In reality, the two lattices will not match exactly. Therefore, the rational number $r_{1} / r_{2}$ will only approximate $A_{2} / A_{1}$. However, prescribing a certain precision to this match will substantially reduce the number of integers $r_{1}$ and $r_{2}$ to be considered.

An upper limit on the possible values of $r_{1}$ and $r_{2}$ is introduced by the requirement that the superlattice unit cell area will not exceed some large prescribed value say $A_{\text {max }}$. In that case, $r_{1} A_{1} \approx r_{2} A_{2}<A_{\max }$, or,

$$
r_{1}<\frac{A_{\max }}{A_{1}}, \quad r_{2}<\frac{A_{\max }}{A_{2}} .
$$

Suppose we have found all those value of $r_{1}$ and $r_{2}$ that satisfy Eq. (2.1) to within a reasonable precision as well as Eq. (2.2). We now have to consider all the possible superlattices of the first lattice that have a unit cell area of $r_{1} A_{1}$ and all the possible superlattices of the second lattice with a unit cell area of $r_{2} A_{2}$, and compare them. In general, given a lattice with a unit cell area $A$, we want to characterize all the superlattices with a unit cell area $n A$, where $n$ is a given integer. We will show that there are only a finite number of such superlattices, not more than the sum of the divisors of $n$. For example, a two-dimensional lattice will have at most 4 distinct superlattices with a unit cell three times larger than that of the original lattice. In this case $4=1+3$ is the sum of the divisors of 3. Similarly, there are at most $12=1+2+3+6$ distinct superlattices with a unit cell six times larger than that of the original lattice.

Suppose we are given a lattice and a superlattice with a unit cell which is $n$ times larger than that of the original lattice. If $(\mathbf{a}, \mathbf{b})$ is a primitive translations set of the original lattice, then one can always find a primitive translations set $(\mathbf{u}, \mathbf{v})$ of the superlattice, satisfying

$$
\left(\begin{array}{l}
\mathbf{u} \\
\mathbf{v}
\end{array}\right)=\left(\begin{array}{cc}
i & j \\
0 & m
\end{array}\right)\left(\begin{array}{l}
\mathbf{a} \\
\mathbf{b}
\end{array}\right)
$$

with $i, j, m$ integers, and

$$
\begin{aligned}
& i \cdot m=n, \\
& i, m>0, \\
& 0 \leqslant j \leqslant m-1 .
\end{aligned}
$$

A formal proof of Eq. (2.3) can be found in Cassels ${ }^{8}$ and will 
not be reproduced here. The number of superlattices to consider, that is the number of different $i, j$, and $m$ satisfying Eqs. (2.4) $-(2.6)$, is reasonably small, and the computer can easily generate them all and compare them to the relevant superlattices of the other lattice.

\section{RESULTS}

To demonstrate the method and explore the role of lattice match in epitaxy, we apply the method for two recently reported interfaces: CdTe on GaAs and CdTe on sapphire. CdTe is considered a good candidate for solar applications, as well as a substrate of $\mathrm{Hg}_{x} \mathrm{Cd}_{\mathbf{i}_{-x}} \mathrm{Te}$ or CdTe-HgTe superlattices for infra red detectors. Due to its bad mechanical properties, it may have to be grown on another material for support. Recently, successful growth of CdTe films on either GaAs (Ref. 1) or sapphire ${ }^{3}$ has been reported

\section{A. CdTe on GaAs}

Both CdTe and GaAs crystalize in the face-centered cubic, zinc-blende structure. The lattice parameters of these crystals are given by $^{4}$

$$
a_{\mathrm{GaAs}}=5.653 \AA, \quad a_{\mathrm{CdTe}}=6.481 \AA .
$$

For both materials, the lattice translations parallel to the (100) face form a grid with square unit cells, $a / \sqrt{2}$ on a side, oriented parallel to the $[01 \overline{1}]$ and $[0 \overline{1} 1]$ directions. The lattice translations parallel to the (110) face form a grid with rectangular cells, $a / \sqrt{2}$ by $a$, whose shorter edge is parallel to the [110] direction and the longer edge parallel to the [001] direction. The lattice translations parallel to the (111) face form a grid made of $60^{\circ}$ rhornbes whose sides are $a / \sqrt{2}$ long

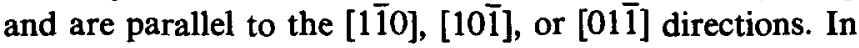
Fig. 3, these grids can be seen as the underlying fine lines. Figure 3 is divided into six regions, showing $\mathrm{CdTe}$ on the left hand and $\mathrm{GaAs}$ on the right hand side, with the (111), (110),

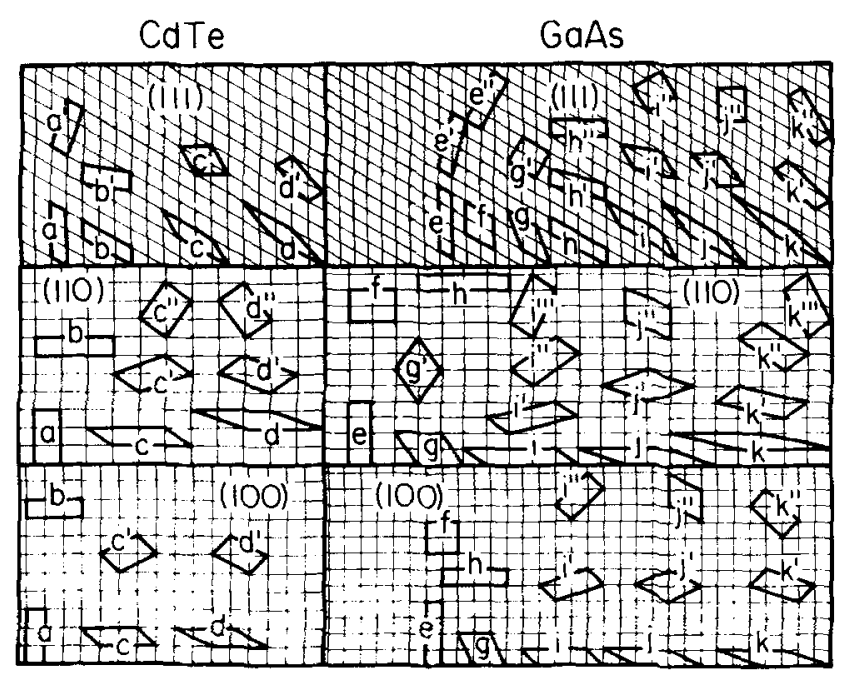

FIG. 3. Lattice translations parallel to the (100), (110), and (111) faces of $\mathrm{CdTe}$ and GaAs. Nonequivalent unit cells of order 3 for CdTe and 4 for $\mathrm{GaAs}$ are shown as parallelograms bounded by thick lines and denoted by unprimed letters. Various stages of the reduction procedure are denoted by primed letters.
TABLE I. Two-dimensional unit cell areas of GaAs and CdTe unreconstructed surfaces in the three major crystal directions. The unit cell areas are given in units of square angstroms.

\begin{tabular}{cccc}
\hline & $(100)$ & $(110)$ & $(111)$ \\
\hline Unit cell area & $a^{2} / 2$ & $a^{2} / \sqrt{2}$ & $a^{2} \frac{\sqrt{3}}{1}$ \\
CdTe, $a=6.481 \AA$ & 21.00 & 29.70 & 18.19 \\
GaAs, $a=5.653 \AA$ & 15.98 & 22.60 & 13.84 \\
\hline \hline
\end{tabular}

and (100) faces of each material from top to bottom. All the grids in Fig. 3 are drawn to the same scale, the whole figure being 200 by $150 \AA$.

It is worth mentioning here that the choice of grids with square cells for $(100)$ or rectangular cells for $(110)$, etc., is by no means unique. This choice amounts to a particular choice of a primitive set of translations for each lattice. Had we picked a different set of primitive translations, the shape of the unit cells would be different, but nevertheless, their areas would be the same. The unit cell areas of the grids appearing in Fig. 3 is summarized in Table 1. Next, we form the ratios $A_{2} / A_{1}$ of Eq. (2.1). Here, $A$, is the unit cell area of the twodimensional lattice formed by the translations of the GaAs, parallel to some given face, and $A_{2}$ is the same for CdTe. The corresponding faces need not be the same for GaAs and CdTe. A partial list of these ratios is given in Table II. We concentrate here only on the three most important faces, namely, the (100), (110), and (111) for each one of the GaAs and the $\mathrm{CdTe}$. This will give us nine possible combinations of area ratios, as can be seen in Table II. The unit cell areas for each row and column were taken from the corresponding entries in Table I. Let us concentrate further only on the main diagonal of Table II. This diagonal corresponds to $(100) /(100),(110) / 110)$, and $(111) /(111)$. The unit cells area ratios are all equal to

$$
\left(\frac{a_{\text {CdTe }}}{a_{\text {GaAs }}}\right)^{2}=1.314
$$

This ratio can be approximated by rational numbers according to Eq. (2.1). A few of the reasonably accurate rational approximations are given by $4 / 3,13 / 10,17 / 13,21 / 16$, etc., with relative errors of $1.4 \%, 1.1 \%, 0.5 \%$, and $0.1 \%$, respectively. It is clear that we may approximate this ratio to arbi-

TABLE II. Ratios of unit cell areas of GaAs and CdTe surfaces in the three major crystal directions. The rational approximations to these ratios give us

\begin{tabular}{|c|c|c|c|c|}
\hline GaAs & $\begin{array}{c}\text { CdTe } \\
\text { Cell area }\end{array}$ & $\begin{array}{c}(100) \\
21.00 \dot{A}^{2}\end{array}$ & $\begin{array}{c}(110) \\
29.70 \dot{A}^{2}\end{array}$ & $\begin{array}{c}(111) \\
18.19 \dot{A}^{2}\end{array}$ \\
\hline$(100)$ & $15.98 \AA^{2}$ & $\frac{21.00}{16.10}=1.314$ & $\frac{2979}{1561}=1.859$ & $\frac{1218}{15.91}=1.138$ \\
\hline (110) & $22.60 \dot{A}^{2}$ & $\frac{21.00}{22100}=.9294$ & $\frac{2970}{22100}=1.314$ & $\frac{1819}{22.105}=.8049$ \\
\hline (111) & $13.84 \dot{A}^{2}$ & $\frac{21.00}{1204}=1.517$ & $\frac{3970}{13}=2.146$ & $\frac{1810}{13.4}=1.314$ \\
\hline
\end{tabular}
the possible combinations of superlattice cell sizes. 
trary precision, if we allow $r_{1}$ and $r_{2}$ of Eq. (2.1) to be arbitrarily large. However, if we restrict the size of $r_{1}$ and $r_{2}$, there are only a limited number of such $r_{1}$ and $r_{2}$ that will approximate the ratio, and the relative error might not be small. Restricting the size of $r_{1}$ and $r_{2}$ is equivalent to restricting the size of the common unit cell area.

Even when we can pick a pair integers $r_{1}$ and $r_{2}$ satisfying Eq. (2.1) to within a good precision, we do not always have a good lattice match. To see this, let us concentrate further on the approximation $4 / 3$ to the ratio $\mathrm{Eq}$. (3.1). We will scan all the superlattices of order 3 in the CdTe and all the superlattices of order 4 in the GaAs, looking for a match. (The order of a superlattice is the area ratio of its unit cell to that of the original lattice. For example, a superlattice of order three has a unit cell area which is three times that of the original lattice.) Such a search for superlattices will be done for each one of the three faces. To find all such superlattices, we apply the transformations Eq. (2.3) to the primitive translations of the original lattice. The results of these transformations on the unit cells is seen in Fig. 3. In general there are four such transformation matrices when $n=3$, namely,

$$
(a)=\left(\begin{array}{ll}
1 & 0 \\
0 & 3
\end{array}\right),(b)=\left(\begin{array}{ll}
3 & 0 \\
0 & 1
\end{array}\right),(c)=\left(\begin{array}{ll}
3 & 1 \\
0 & 1
\end{array}\right),(d)=\left(\begin{array}{ll}
3 & 2 \\
0 & 1
\end{array}\right),
$$

and seven matrices when $n=4$, namely,

$$
\begin{aligned}
& (e)=\left(\begin{array}{ll}
1 & 0 \\
0 & 4
\end{array}\right),(f)=\left(\begin{array}{ll}
2 & 0 \\
0 & 2
\end{array}\right),(g)=\left(\begin{array}{ll}
2 & 1 \\
0 & 2
\end{array}\right),(h)=\left(\begin{array}{ll}
4 & 0 \\
0 & 1
\end{array}\right), \\
& (i)=\left(\begin{array}{ll}
4 & 1 \\
0 & 1
\end{array}\right),(j)=\left(\begin{array}{ll}
4 & 2 \\
0 & 1
\end{array}\right),(k)=\left(\begin{array}{ll}
4 & 3 \\
0 & 1
\end{array}\right) .
\end{aligned}
$$

After one of the transformations of Eq. (2.3), or in our case Eq. (3.2) or (3.3), is applied to the unit cell, a larger cell is obtained. In this case, three times larger for $\mathrm{CdTe}$ and four times larger for GaAs. From these unit cells we can form superlattices by juxtaposing copies of the same cell. Moreover, every superlattice with unit cell area three times larger than that of the original lattice will have a unit cell that is given by one of $(a)-(d)$. Therefore the cells (a)-(d) generate all the possible distinct superlattices of order 3 . The same is true for the cells (e)-(k) with regard to superlattices of order 4 . The result of the application of each one of the transformations of Eq. (3.2) to the standard unit cell of the $\mathrm{CdTe}(100)$, $\mathrm{CdTe}(110)$, and $\mathrm{CdTe}(111)$ faces is seen in the left side of Fig. 3 . These new cells are denoted by the same letter as the corresponding transformation in Eq. (3.2). Similarly, the application of the transformations in Eq. (3.3) to the unit cell of the three GaAs faces is seen in the right side of the same figure and denoted by (e)-(k). Each one of the transformations in Eq. (3.2) and (3.3), when applied to the original unit cell, results in a particular shape of a superlattice unit cell. These larger unit cells are by no means unique, and the reduction scheme, described in the previous section, is applied. The reduction scheme consists of successive vector additions or subtractions of the shorter side of the cell from the longer until no further reduction in length is possible. The reduction steps for each one of the (a)-(k) cells are shown by primed letters in Fig. 3. For example, the cell (a) near the upper left corner of Fig. 3 corresponds to the matrix $(a)$ of Eq. (3.2) applied to the unit cell of the $\mathrm{CdTe}(111)$ face. If we subtract the shorter side of this cell, taken as a vector, from the longer side, we get the cell $\left(\mathrm{a}^{\prime}\right)$. In the figure, we have moved (a) up and away from (a) for clarity, but they should have shared a common side. One can see that the long side of the new cell $\left(a^{\prime}\right)$ is shorter than the long side of (a). If we repeat this procedure once again, the longer side of the new cell will not be shorter than the longer side of (a). We must conclude that the cell $\left(\mathrm{a}^{\prime}\right)$ is reduced. Similarly, one can apply the reduction scheme to any one of the other cells $(b)-(k)$. For the particular cells appearing in Fig. 3, after at most three reduction steps, all the cells are reduced. One should keep in mind that all the different cells generated during the reduction process are unit cells of the same superlattice. For example, suppose we generate two superlattices of the CdTe(111) by juxtaposing an infinite number of copies of either one of (a) or $\left(a^{\prime}\right)$. These two superlattices might appear different only as long as we retain the edges of the cells. However, if we look only at the corners of the cells, the two superlattices are identical.

The purpose of the reduction procedure is to select a particular shape of unit cell among all the possible cells of a given superlattice. After reduction, we can see that, if the original lattice possessed any nontrivial symmetries, some of the larger unit cells are actually equivalent. Thus, in the (100) face of the $\mathrm{CdTe}$, (b) can be obtained from (a) by a $90^{\circ}$ rotation, while $\left(c^{\prime}\right)$ and $\left(d^{\prime}\right)$ are mirror images of each other. The dimensions of the reduced three-fold enlarged CdTe unit cells, as well as those of the reduced four-fold enlarged GaAs unit cells of Fig. 3, appear in Table III. In that table, each row, denoted by (a)....(k), corresponds to a particular transformation matrix of Eqs. (3.2) and (3.3), as well as to the unit cells appearing in Fig. 3. The transformation matrices of Eqs. (3.2) and (3.3) appear in this table in the second column from the left. The rest of Table III is divided into three major columns, corresponding to $\mathrm{CdTe}(100) / \mathrm{GaAs}(100)$, $\mathrm{CdTe}(110) / \mathrm{GaAs}(110)$, and $\mathrm{CdTe}(111) / \mathrm{GaAs}(111)$. Each such major column is subdivided into four columns describing the dimensions of the reduced unit cell. In our notation, $\mathrm{a}, \mathrm{b}$, and $\alpha$ correspond to the shorter side of the cell, the longer side, and the angle between them. Since the structure of both GaAs and CdTe is cubic, these $\mathrm{a}, \mathrm{b}$, and $\cos (\alpha)$ can be expressed as simple algebraic expressions in the lattice parameter (denoted by italic $a$ ). These expressions, together with their numerical values, appear in the body of Table III. We would like to emphasize that the transformation matrices appearing in Table III are those applied to the unit cells before the reduction, i.e., they are the same as Eqs. (3.2)(3.3), while the unit cells dimensions appearing in the same table are those of the cells after reduction. By comparing the dimensions of the reduced unit cells in Table III, we can conclude that there is no reasonable 3:4 match of the (100)/ $(100)$, but there are $3: 4$ matches of $(110) /(110)$, namely, $\left(c^{\prime \prime}\right)$ or (d") to (f), and there is also a 3:4 match of the (111)/(111), namely, $\left(c^{\prime}\right)$ to ( $\left.f\right)$. For the $(110) /(110)$ case, the cells $\left(c^{\prime \prime}\right)$ and $\left(d^{\prime \prime}\right)$ have their short side oriented along a CdTe [111] direction and the long side along a $\mathrm{CdTe}[1 \overline{1} \overline{2}]$ direction (or [1 $1 \overline{1}]$ and [1 12 ], etc.). The ( $\mathrm{f}$ ) cell has its short side along the GaAs [110] direction and its long side along the GaAs[001] direction. For the $(111) /(111)$ case, the $\left(c^{\prime}\right)$ cell has all its sides 
TABLE III. Dimensions of reduced superlattice unit cells for the possible 3:4 lattice match of CdTe(100)/GaAs(100), CdTe(110)/GaAs(110) and CdTe(111)/ GaAs(111). All the possible superlattices of order 3 are obtained from the matrices (a)-(d) operating on the CdTe original unit cell. Likewise, all the possible superlattices or order 4 are obtained from the seven matrices (c)-(k) operating on the GaAs unit cell. After an appropriate reduction is applied to the superlattice cells, their dimensions are displayed in this table. $a$ and $b$ are the reduced cell edges in angstroms, and $\alpha$ is the angle between them, in degrees.

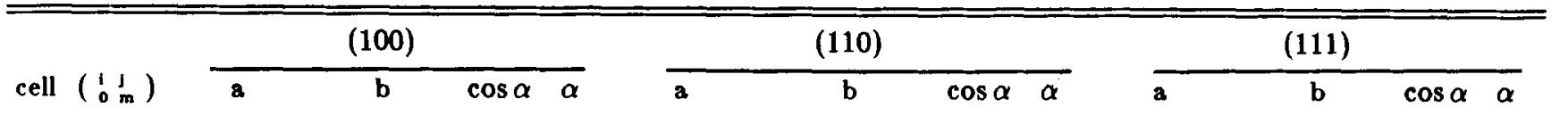

1. CdTe
(a) $\left(\begin{array}{ll}3 & 0 \\ 0 & 1\end{array}\right)$
(b) $\left(\begin{array}{ll}1 & 0 \\ 0 & 3\end{array}\right)$
(c) $\left(\begin{array}{ll}1 & 1 \\ 0 & 3\end{array}\right)$
$\frac{a}{\sqrt{2}}=4.58 \quad \frac{3 a}{\sqrt{2}}=13.75$
$\frac{a}{\sqrt{2}}=4.58 \quad \frac{3 a}{\sqrt{2}}=13.75$
$a=6.48$
$\frac{\sqrt{2}}{\sqrt{2}}=10.25$
$a=6.48 \quad \frac{a \sqrt{5}}{\sqrt{2}}=10.25$
(d) $\left(\begin{array}{ll}1 & 2 \\ 0 & 3\end{array}\right)$
0
0
$\frac{1}{\sqrt{10}}$
2. GaAs

$\begin{array}{ll}90.0 & a=6.481 \\ 90.0 & \frac{a}{\sqrt{2}}=4.58 \\ 71.6 & \frac{a \sqrt{3}}{\sqrt{2}}=7.94 \\ 71.6 & \frac{a \sqrt{3}}{\sqrt{2}}=7.94\end{array}$

$\frac{3 a}{\sqrt{2}}=13.75$

$3 a=19.44$

$a \sqrt{3}=11.22$

$a \sqrt{3}=11.22$
0
0

090

90.0

$\frac{a}{\sqrt{2}}=4.58$

$\frac{a \sqrt{7}}{\sqrt{2}}=12.12$

$\frac{1}{2 \sqrt{7}}$

90.0

$90.0 \frac{a}{\sqrt{2}}=4.58$

$\frac{a \sqrt{7}}{\sqrt{2}}=12.12 \frac{1}{2 \sqrt{7}}$

$\frac{1}{\sqrt{7}} 79.1$

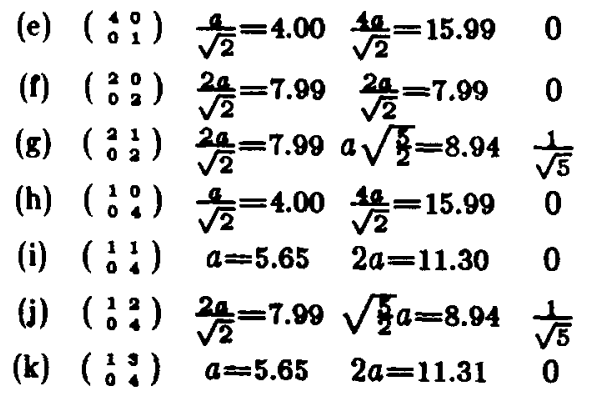

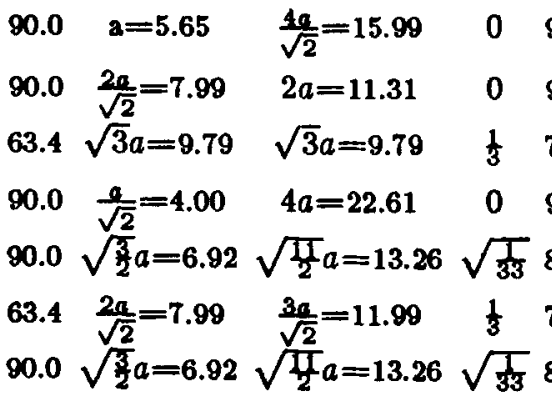

$90.0 \quad \frac{a}{\sqrt{2}}=4.00$
$90.0 \quad \frac{2 a}{\sqrt{2}}=7.99$
$70.5 \quad \sqrt{\frac{3}{2}} a=6.92$
$90.0 \quad \frac{a}{\sqrt{2}}=4.00$

$90.0 \quad \frac{a}{\sqrt{2}}=4.00 \quad \sqrt{6} a=13.85$

$80.0 \sqrt{\frac{3}{2}} a=6.92$

$70.5 \sqrt{\frac{3}{2}} a=6.92$

80.0

$\frac{a}{\sqrt{2}}=4.00 \quad \sqrt{6} a=13.85$ 
TABLE IV. Good lattice matches of CdTe on GaAs. The primitive common unit cells in this table do not exceed $400 \AA^{2}$, and the mismatch is less than $1 \%$. Under these conditions, all the possible matches of $\mathrm{CdTe}(100),(110)$, and (111) on $\mathrm{GaAs}(100),(110)$ and (111) are given in this table. For each possible match, we give here the epitaxial condition, as well as the common unit cell dimensions on each side of the interface. The epitaxial condition is a pair of crystal directions, one on each side of the interface, that will be parallel to each other. Many such pairs are possible, and only one of them is given here. The cells' dimensions in angstroms and degrees are given here for comparison. The mismatch percentage in all the three dimensions of the common unit cell is given in the last three columns.

\begin{tabular}{|c|c|c|c|c|c|c|c|c|c|c|c|}
\hline \multirow{2}{*}{$\begin{array}{l}\text { Matching faces } \\
C d T e / G a A s\end{array}$} & \multirow{2}{*}{$\begin{array}{l}\text { Epitaxial condition } \\
\qquad C d T e \| G a A s\end{array}$} & \multirow{2}{*}{$\begin{array}{l}\text { Cell area } \\
\left(\dot{A}^{2}\right)\end{array}$} & \multicolumn{3}{|c|}{$C d T e$} & \multicolumn{3}{|c|}{ GaAs } & \multirow[b]{2}{*}{$\%_{a}$} & \multirow[b]{2}{*}{$\%_{b b}$} & \multirow[b]{2}{*}{$\%_{\alpha}$} \\
\hline & & & $\bar{a}$ & $b$ & $\alpha$ & $\bar{a}$ & $\bar{b}$ & $\alpha$ & & & \\
\hline$(100) /(100)$ & {$[012] \|[015]$} & 210 & 14.43 & 14.49 & 90.00 & 14.41 & 14.41 & 90.00 & 0.5 & 0.5 & 0.0 \\
\hline$(100) /(100)$ & {$[015] \|[035]$} & 273 & 16.52 & 16.52 & 90.00 & 16.48 & 16.48 & 90.00 & 0.3 & 0.3 & 0.0 \\
\hline$(111) /(100)^{\mathrm{a}}$ & {$[1 \overline{2} 1] \|[011]^{\mathrm{a}}$} & 127 & 7.938 & 16.52 & 76.10 & 7.995 & 16.48 & 75.96 & 0.7 & 0.3 & 0.2 \\
\hline$(100) /(110)$ & {$[011] \|[1 \overline{1} 2]$} & 315 & 13.75 & 23.37 & 78.69 & 13.85 & 23.31 & 78.58 & 0.7 & 0.3 & 0.1 \\
\hline$(100) /(110)$ & {$[015] \|[3 \overline{3} 4]$} & 315 & 16.52 & 19.44 & 78.69 & 16.48 & 19.58 & 78.58 & 0.3 & 0.7 & 0.1 \\
\hline$(110) /(110)$ & {$[\overline{1} 12] \|[1 \overline{1} 0]$} & 89 & 7.938 & 11.22 & 90.00 & 7.995 & 11.31 & 90.00 & 0.7 & 0.7 & 0.0 \\
\hline$(111) /(111)$ & {$[1 \overline{2} 1] \|[1 \overline{1} 0]$} & 55 & 7.938 & 7.938 & 60.00 & 7.995 & 7.995 & 60.00 & 0.7 & 0.7 & 0.0 \\
\hline$(111) /(111)$ & {$[1 \overline{1} 0] \|[5 \overline{4} \overline{1}]$} & 291 & 18.33 & 18.33 & 60.00 & 18.32 & 18.32 & 60.00 & 0.1 & 0.1 & 0.0 \\
\hline$(111) /(111)$ & {$[23 \overline{5}] \|||[1 \overline{1} 0]$} & 346 & 19.98 & 19.98 & 60.00 & 19.99 & 19.99 & 60.00 & 0.1 & 0.1 & 0.0 \\
\hline$(111) /(111)$ & {$[1 \overline{5} 4] \|[1 \overline{3} 2]$} & 382 & 21.00 & 21.00 & 60.00 & 21.15 & 21.15 & 60.00 & 0.7 & 0.7 & 0.0 \\
\hline
\end{tabular}

${ }^{a}$ Observed experimentally by J. T. Cheung (Ref. 1.)

TABLE V. Good lattice matches of CdTe on sapphire. The primitive common unit cells in this table do not exceed $600 \AA^{2}$, and the mismatch is less than $1 \%$. Under these conditions, all the possible matches of $\mathrm{CdTe}(100),(110),(111),(210),(211)$ or $(221)$ on $A l_{2} O_{3}(101),(011)$ or $(111)$ are given here. The sapphire faces and directions in this table refer to the rhomobohedral notation. Transformations from rhombohededral to hexagonal notations are given in the appendix. The meaning of the columns in this table are identical to those of Table IV.

\begin{tabular}{|c|c|c|c|c|c|c|c|c|c|c|c|}
\hline \multirow{2}{*}{$\begin{array}{l}\text { Matching faces } \\
\mathrm{CdTe} / \mathrm{Al}_{2} \mathrm{O}_{3}\end{array}$} & \multirow{2}{*}{$\begin{array}{l}\text { Epitaxial condition } \\
\qquad \mathrm{CdTe} \| \mathrm{Al}_{2} \mathrm{O}_{3}\end{array}$} & \multirow{2}{*}{$\begin{array}{c}\text { Cell area } \\
\left(\dot{A}^{2}\right)\end{array}$} & \multicolumn{3}{|c|}{$C d T e$} & \multicolumn{3}{|c|}{$\mathrm{Al}_{2} \mathrm{O}_{3}$} & \multirow[b]{2}{*}{$\%_{a}$} & \multirow[b]{2}{*}{$\% b$} & \multirow[b]{2}{*}{$\%_{\alpha}$} \\
\hline & & & $\bar{a}$ & $b$ & $\bar{\alpha}$ & $\bar{a}$ & $\bar{b}$ & $\bar{\alpha}$ & & & \\
\hline$(110) /(101)$ & {$[111] \|[121]$} & 505 & 11.22 & 45.13 & 85.24 & 11.31 & 45.51 & 84.87 & 0.7 & 0.8 & 0.4 \\
\hline$(110) /(101)$ & {$[3 \overline{3} 2] \|[31 \overline{3}]$} & 564 & 15.20 & 37.23 & 85.74 & 15.17 & 37.14 & 85.09 & 0.2 & 0.2 & 0.8 \\
\hline$(111) /(101)$ & {$[4 \overline{5} 1] \|[14 \overline{1}]$} & 509 & 21.00 & 24.25 & 90.00 & 21.06 & 24.34 & 89.10 & 0.3 & 0.4 & 1.0 \\
\hline$(210) /(101)$ & {$[1 \overline{2} \overline{5}] \|[3 \overline{2} \overline{3}]$} & 564 & 17.75 & 31.75 & 90.00 & 17.58 & 31.94 & 89.10 & 1.0 & 0.6 & 1.0 \\
\hline$(211) /(101)$ & {$[1 \overline{1} \overline{1}] \|[12 \overline{1}]$} & 412 & 11.22 & 36.66 & 90.00 & 11.31 & 36.70 & 89.90 & 0.7 & 0.1 & 0.1 \\
\hline$(100) /(0 \overline{1} 1)$ & {$[013] \|[100]$} & 357 & 10.25 & 34.90 & 86.63 & 10.26 & 34.84 & 87.42 & 0.1 & 0.2 & 0.9 \\
\hline$(100) /(0 \overline{1} 1)$ & {$[011] \|[011]$} & 504 & 9.166 & 54.99 & 90.00 & 9.086 & 55.00 & 89.25 & 0.9 & 0.0 & 0.8 \\
\hline$(110) /(0 \overline{1} 1)$ & {$[001] \|[111]$} & 535 & 12.96 & 41.24 & 90.00 & 12.99 & 41.22 & 90.00 & 0.2 & 0.1 & 0.0 \\
\hline$(110) /(0 \overline{1} 1)$ & [1īo] || [011] & 535 & 9.166 & 58.51 & 85.51 & 9.086 & 59.07 & 86.10 & 0.9 & 1.0 & 0.7 \\
\hline$(111) /(0 \overline{1} 1)$ & {$[01 \overline{1}] \|[011]$} & 327 & 9.166 & 35.79 & 86.33 & 9.086 & 35.44 & 86.10 & 0.9 & 1.0 & 0.3 \\
\hline$(221) /(0 i 1)$ & {$[1 \overline{10}] \|[011]$} & 504 & 9.166 & 54.99 & 90.00 & 9.086 & 55.00 & 89.25 & 0.9 & 0.0 & 0.8 \\
\hline$(100) /(111)$ & {$[051] \|[2 \overline{1} \overline{1}]$} & 546 & 16.52 & 33.05 & 90.00 & 16.49 & 33.31 & 90.00 & 0.2 & 0.8 & 0.0 \\
\hline$(100) /(111)$ & {$[053] \|[01 \overline{1}]$} & 546 & 18.89 & 28.98 & 85.60 & 19.04 & 28.95 & 85.28 & 0.7 & 0.1 & 0.4 \\
\hline$(111) /(111)$ & {$[31 \overline{4}] \|[1 \overline{2} 1]$} & 236 & 16.52 & 16.52 & 60.00 & 16.49 & 16.49 & 60.00 & 0.2 & 0.2 & 0.0 \\
\hline$(111) /(111)$ & {$[1 \overline{2} 1] \|[1 \overline{1} 0]$} & 491 & 23.81 & 23.81 & 60.00 & 23.80 & 23.80 & 60.00 & 0.1 & 0.1 & 0.0 \\
\hline
\end{tabular}


unit cell with $a=4.759$ and $c=12.991$. While this common practice may have some advantages, ${ }^{1}$ it is not recommended for lattice match. ${ }^{12}$ Therefore, we refer to the rhombohedral notation, and the Appendix will clarify the transformation from rhombohedral to hexagonal notation. The sapphire (101), $(0 \overline{1} 1)$, and (111) faces correspond to the $(1 \overline{1} 02),(1 \overline{2} 10)$, and (0001) faces in the hexagonal notation. Out of the 18 possible face combinations, only 10 can match to within $1 \%$, with a superlattice cell area not exceeding $600 \AA^{2}$. These matches are shown in Table $V$, which is structured like Table IV. As one can see, the CdTe(111) matches all the three sapphire planes reported by Myers et $a l .{ }^{3}$ Since they do not provide any further information about the orientation of the film with respect to the substrate, further experiments are needed to confirm or reject this theory.

\section{SUMMARY AND DISCUSSION}

The theory of geometrical lattice match was formalized in this paper. This theory is applicable to any pair of crystal structures and for any direction. We defined two lattices to match if the two 2D lattices, formed by the crystal translations parallel to the interface, have a common superlattice. In general, we cannot hope to achieve a perfect match, and instead of a common superlattice, we have a pair of superlattices, one for each side of the interface, that are almost identical. There are two parameters that characterize the match: the mismatch between the two superlattices and the unit cell area of these superlattices. We have shown how to obtain a standard unit cell for a given lattice. This unit cell is a parallelogram with sides $a$ and $b$ and an acute angle $\alpha$. By comparing $a, b$, and $\alpha$ for both superlattices, we obtain the mismatch between them. The unit cell area can be taken from either one of the two superlattices, since they are almost equal. We have developed a constructive way to find all the possible lattice matches with a given mismatch and common unit cell area smaller than a prescribed value.

The role of geometrical match in epitaxy is still not clear, since local interface chemistry will always play a major role. Therefore, if two lattices match, it does not mean that they will grow epitaxially on each other. We do hope, however, to establish that a thick epitaxial growth having a sharp periodic interface is possible only when there is a good match. We have shown that this is indeed the case for the reported cases of epitaxial growth of $\mathrm{CdTe}(111)$ on $\mathrm{GaAs}(100),{ }^{1} \mathrm{Al}_{2} \mathrm{O}_{3}(101), \mathrm{Al}_{2} \mathrm{O}_{3}(1 \overline{1} 0)$, and $\mathrm{Al}_{2} \mathrm{O}_{3}(111){ }^{3}$ For each one of these cases, a common unit cell area of $600 \AA^{2}$ was large enough, and the resulting mismatch was less than $1 \%$.

Further theoretical and experimental investigation is required in order to establish general criteria for allowable unit cell areas and mismatch.

\section{ACKNOWLEDGMENTS}

We would like to acknowledge fruitful discussions with Professor Marc A. Nicolet, who first inspired us to think in these directions. This work was supported in part by the office of Naval Research under contract No. N00014-79-C0797.

\section{APPENDIX: RHOMBOHEDRAL AND HEXAGONAL NOTATION}

When one works with rhombohedral materials like sapphire, it is sometimes convenient to transform to hexagonal notations. We will follow the conventions of M. L. Kronberg. ${ }^{13}$

Three rhombohedral unit cells are joined to give a hexagonal unit cell. If $\mathbf{a}_{1}, \mathbf{a}_{2}$ and $\mathbf{a}_{3}$ are the rhombohedral primitive vectors, one can form a set of hexagonal vectors $\mathbf{A}_{1}, \mathbf{A}_{2}$, $\mathbf{A}_{3}$, and $\mathbf{C}$ such that

$$
\begin{aligned}
& A_{1}=a_{1}-a_{2}, \\
& A_{2}=a_{2}-a_{3}, \\
& A_{3}=a_{3}-a_{1}, \\
& C=a_{1}+a_{2}+a_{3} .
\end{aligned}
$$

The inverse of this transformation is given by

$$
\begin{aligned}
& \mathbf{a}_{1}=\frac{2 \mathbf{A}_{1}+\mathbf{A}_{2}+\mathbf{C}}{3}, \\
& \mathbf{a}_{2}=\frac{-\mathbf{A}_{1}+\mathbf{A}_{2}+\mathbf{C}}{3}, \\
& \mathbf{a}_{3}=\frac{-\mathbf{A}_{1}+2 \mathbf{A}_{2}+\mathbf{C}}{3} .
\end{aligned}
$$

If $\mathbf{v}=\alpha \mathbf{a}_{1}+\beta \mathbf{a}_{2}+\gamma \mathbf{a}_{3}$, is a vector in thombohedral coordinates, then the hexagonal representation is

$$
\mathbf{v}=\frac{2 \alpha-\beta-\gamma}{3} \mathbf{A}_{1}+\frac{\alpha+\beta-2 \gamma}{3} \mathbf{A}_{2}+\frac{\alpha+\beta+\gamma}{3} \mathbf{C} \text {. }
$$

Thus,

$$
\begin{aligned}
& {[\alpha, \beta, \gamma](R)=\left[\frac{\alpha-\beta}{3}, \frac{\beta-\gamma}{3}, \frac{\gamma-\alpha}{3}, \frac{\alpha+\beta+\gamma}{3}\right](H)} \\
& {[\text { e.g., }[1,1,0](R)=[0,1, \overline{1}, 2](H)] .}
\end{aligned}
$$

Similarly for reciprocal lattices, if $\left(\mathbf{b}_{1}, \mathbf{b}_{2}, \mathbf{b}_{3}\right)$ are the primitive translations of the reciprocal rhombohedral lattice, and $\left(\mathbf{B}_{1}, \mathbf{B}_{2}, \mathbf{B}_{3}\right)$ are the translations of the reciprocal hexagonal lattice, then

$$
\begin{aligned}
& \mathbf{b}_{1}=\mathbf{B}_{1}+\mathbf{B}_{3}, \\
& \mathbf{b}_{2}=-\mathbf{B}_{1}+\mathbf{B}_{2}+\mathbf{B}_{3}, \\
& \mathbf{b}_{3}=-\mathbf{B}_{2}+\mathbf{B}_{3},
\end{aligned}
$$

and the $(\alpha, \beta, \gamma)$ face (rhombohedral notation) is the same as the $(\alpha-\beta, \beta-\gamma, \gamma-\alpha, \alpha+\beta+\gamma)$ (hexagonal notation [e.g., $(110)(R)=(01 \overline{1} 2)(H),(411)(R)=(30 \overline{3} 6)=(10 \overline{1} 2)(H)]$.

The relationships between the hexagonal parameters $\left(a_{h}, c\right)$ and the rhombohedral parameters $\left(a_{r}, \alpha\right)$ are given by ${ }^{14}$

$$
\begin{aligned}
& a_{r}=\sqrt{a_{h}^{2} / 3+c^{2} / 9}, \cos \alpha=\frac{-a_{h}^{2} / 2+c^{2} / 3}{a_{h}^{2}+c^{2} / 3}, \\
& a_{h}=2 a_{r} \sin \frac{\alpha}{2}, c=3 \sqrt{a_{r}^{2}-a_{h}^{2} / 3} .
\end{aligned}
$$

'J. T. Cheung, Proceedings of the 1983 U.S. Workshop on the Physics and Chemistry of Mercury Cadmium Telluride, p. 13.

${ }^{2} \mathrm{G}$. W. Cullen, in Heteroepitaxial Semiconductors for Electronic Devices, edited by G. W.Cullen and C. C. Wang (Springer, New York, 1978), p. 50. ${ }^{3}$ T. H. Myers, Y. Cheng, R. N. Bicknell, and J. F. Schetzina, Appl. Phys. Lett. 42, 247 (1983).

${ }^{4}$ J. D. H. Donnay and H. M. Ondik, Crystat Data, Determinative Tables, 
3rd ed. (U. S. Department of Commerce, National Bureau of Standards and Joint Commission on Powder Diffraction Standards, Washington, 1973), Vol II.

${ }^{5} \mathrm{G}$. Friedel, Lecons de Cristallographie, (Berger Levrault, Paris, 1926).

${ }^{6}$ P. H. Pumphrey, in Grain Boundary Structure and Properties, edited by G.

A. Chadwick and D. A. Smith (Academic, London, 1976), p.139.

'S. Ranganathan, Acta Cryst. 21, 197 (1966).

${ }^{8}$ J. W. S. Cassels, An Introduction to the Geometry of Numbers (Springer, Berlin, 1959).

${ }^{9}$ A. Santoro and A. D. Mighell, Acta Cryst. A 29, $171\{1973\}$

${ }^{10} \mathrm{M}$. Buerger, Elementary Crystallography (MIT, Cambridge, 1978), p. 106.
"J. L. Schmit (private communication).

12The main disadvantages are that using hexagonal instead of rhombohedral notation arbitrarily increases every unit unit cell by a factor of $1-3$. It also creates ambiguity in the notation. For example, the hexagonal [1 1102 ) and $(\overline{1} 102)$ are not equivalent. One corresponds to the rhombohedral (101), the other to [141], but there is no way to tell which one is which without knowing whether the hexagonal $\left\{\mathbf{a}_{1}, \mathbf{a}_{2}, \mathbf{c}\right)$ axes form a right or left hand system.

${ }^{13}$ M. L. Kronberg, Acta Met. 5, 507 (1957).

${ }^{14} \mathrm{~J}$. C. Slater, Symmetry and Energy Bands in Crystals (Dover, New York, 1972),p. 21. 